\title{
Estudo da utilização de materiais de referência nas análises de água por laboratórios envolvidos no sistema de acreditação
}

\author{
Suzana Saboia de Moura UFF \\ Stella Regina Reis da Costa UFF
}

\section{RESUMO}

O presente estudo propõe-se discutir o uso de materiais de referência (MR) pelos laboratórios de ensaios de águas, sob o ponto de vista da aquisição dos materiais conforme o item "Aquisição de serviços e suprimentos" (4.6) da norma ABNT NBR ISO/IEC 17025. 0 papel dos MR nas análises químicas é fornecer referências metrológicas visando assegurar a rastreabilidade das medições. A rastreabilidade é um aspecto essencial da garantia da qualidade para se obter aceitação de dados analíticos nos mercados nacional e internacional. Neste estudo de caso, de caráter exploratório e qualitativo, uma pesquisa foi realizada junto a todos os laboratórios de ensaios prestadores de serviços analíticos para a Agência Nacional de Águas (ANA) e envolvidos no sistema de acreditação pela Coordenação Geral de Acreditação do Inmetro (Cgcre/Inmetro), utilizando-se questionário estruturado e com 50\% de retorno. Os resultados mostraram que são muito poucos os certificados de MR com reconhecimento de terceira parte, ou seja, por organismos de acreditação, segundo normas consagradas que tratam efetivamente da rastreabilidade metrológica. Propõe-se, finalmente, uma política para garantir, sempre que possível, a rastreabilidade dos resultados de ensaios dos laboratórios, na ausência de MR certificados.

\section{Case study on the usage of reference materials in water analysis by laboratories involved in the accreditation system}

\section{ABSTRACT}

This paper aims at discussing the use of reference materials (RM) by water testing laboratories, on the context of RM purchase, based on the requirements of the standard ABNT NBR ISO/IEC 17025, section "Purchasing services and supplies" (4.6). The role of RM in chemical analysis is to provide metrological reference to assure measurement traceability. This is an essential aspect of quality assurance in order to have analytical results accepted in the international market. In this case study, based on the methodological exploratory and qualitative approach, a questionnaire was sent to testing laboratories which provide analytical services to the National Agency for Waters involved in the accreditation system performed by the General Coordination for Accreditation of Inmetro (Cgcre/Inmetro) by using structured questionnaire and with 50\% of return. The results showed that there are few RM certificates with indication of third party recognition, i.e., accreditation bodies, based on standards dealing with metrological traceability. Finally, a policy is proposed to assure the traceability of laboratories measurement results, when certified RMs are unavailable.

\section{KEY WORDS}

Water analysis, traceability, reference material. 


\section{INTRODUÇÃO}

O Governo Brasileiro, reconhecendo a importância da sustentabilidade, que também está relacionada à gestão de seus recursos hídricos, assinou a Carta de Princípios do Rio (Rio-92) e adotou as suas orientações. A criação da Agência Nacional de Águas (ANA) é uma das ações decorrentes da política de gestão dos recursos hídricos.

Em março de 2005, a ANA lançou o Programa Nacional de Acreditação de Laboratórios em Análise da Qualidade da Água (PROLAB). Foi assinado com a Coordenação Geral de Acreditação do Inmetro (Cgcre/Inmetro) um termo de cooperação, que objetiva implantar uma rede de laboratórios acreditados que forneça o suporte legal para as ações de regulação do uso da água e de monitoramento da qualidade das águas interiores em todo o Território Nacional. O PROLAB identifica uma série de parâmetros físico-químicos e químicos, indicadores de matéria orgânica, biológicos e microbiológicos, e elementos e substâncias potencialmente prejudiciais considerados prioritários para o controle da qualidade da água, dispostos pela Resolução CONAMA n. ${ }^{0}$ 357, de 17 de março de 2005.

Considerando-se essa necessidade, é preciso também atender à crescente demanda por resultados de medição de procedência conhecida e aceitável, com rastreabilidade metrológica comprovada. Para atingir esses objetivos, é necessário o uso de elementos de referência - padrões rastreáveis e/ou materiais de referência - na calibração e validação dos métodos de medição. Sem eles é impossível estabelecer uma cadeia segura de rastreabilidade. A rastreabilidade é um aspecto essencial da garantia da qualidade para se obter aceitação internacional de dados analíticos.

Se vários laboratórios conseguem atingir o mesmo resultado analítico para um mesmo material de referência certificado, isto mostra que as suas medições podem ser comparáveis (ISO, 2000).

No Brasil, a Cgcre/Inmetro é o organismo de acreditação que opera um sistema de acreditação de laboratórios reconhecido por seus pares signatários do Acordo de Reconhecimento Mútuo da International Laboratory Accreditation Cooperation (ILAC), do Acordo Bilateral com a European Co-operation for Accreditation (EA) e do Acordo Multilateral com a Interamerican Accreditation Cooperation (IAAC). Unger (2001) explica que o Acordo da ILAC tem o objetivo de desenvolver uma rede global de laboratórios de ensaio e de calibração acreditados, com resultados de medições confiáveis e exatos. O Acordo proporciona uma infra-estrutura técnica ao comércio internacional, promovendo a confiança e aceitação além fronteiras, pelas partes envolvidas, de dados de ensaios e de calibração, ou, em outras palavras, "testado uma vez, aceito em qualquer lugar". A acreditação é definida como "atestação realizada por terceira parte relativa a um organismo de avaliação da conformidade, exprimindo demonstração formal de sua competência para realizar tarefas específicas de avaliação de conformidade" (ABNT NBR ISO/ IEC 17000:2005, p.5).

\section{$\Delta$ rastreabilidade é um aspecto essencial da garantia da qualidade para se obter aceitação internacional de dados analíticos.}

Em obediência às diretrizes do Acordo mencionado, a Cgcre/Inmetro adota a norma ISO/IEC 17025 no reconhecimento de competência técnica de laboratórios, publicada no País pela Associação Brasileira de Normas Técnicas (ABNT) como ABNT NBR ISO/IEC 17025. Essa norma dá especial importância à demonstração da rastreabilidade da medição, que inclui o uso de materiais de referência. Os materiais são aplicados na calibração e na validação de métodos, no controle da qualidade e na verificação do uso correto de um método (Inmetro, 2005). No Brasil, até onde se conhece, são poucos os materiais de referência certificados produzidos de acordo com as recomendações da ISO (International Organization for Standardization) - ISO Guides 3035. Deve-se ressaltar que a ILAC já decidiu incluir no acordo de reconhecimento mútuo vigente (laboratórios) a acreditação de produtores de materiais de referência, logo que os procedimentos apropriados para essa atividade forem desenvolvidos e acordados. O critério de acreditação será a combinação do ISO Guide 34 e da ISO/IEC 17025 (ILAC, 2004).

Esta pesquisa delimita-se a investigar a utilização de materiais de referência na análise de água, ao invés de estudar a aplicação dos materiais de referência em geral, pois esta se estende a outras áreas de ensaios químicos e os materiais são de matrizes variadas. A importância da qualidade da água e da preservação dos recursos hídricos para o Brasil é indiscutível, visto que o nosso País detém 13,7\% da água doce superficial do mundo (ANA, 2005).

Dessa forma, este artigo propõe-se a discutir a utilização de materiais de referência pelos laboratórios de análises de água. O universo pesquisado compreendeu os laboratórios que atuam na análise de águas, sejam eles acreditados ou em fase de acreditação pela Cgcre/Inmetro, além de outros laboratórios não acreditados que também prestam serviços analíticos para a ANA e que participaram de uma comparação interlaboratorial organizada pela Diretoria de 
Metrologia Científica e Industrial (Dimci) do Inmetro, que visou complementar a atuação do Inmetro na parceria com a ANA. Dessa forma, a pesquisa visou analisar as respostas de todos os laboratórios utilizados pela ANA, já que o principal foco era obter informações sobre a garantia da rastreabilidade metrológica dos resultados de medição e, consequentemente a confiabilidade dos relatórios de ensaio utilizados pela Agência.

\section{REFERENCIAL TEÓRICO}

A harmonização de normas e regulamentos técnicos é fundamental para as relações comerciais, tanto em âmbito internacional quanto nacional, além da necessidade de se investir em procedimentos que visem à garantia da qualidade dos produtos comercializados. Desta forma, visando a garantir a qualidade dos produtos, cabe a inserção de outros dois temas de suma importância ao comércio internacional: a metrologia e a avaliação da conformidade, formando o alicerce para qualquer sistema aplicado à promoção das relações comerciais, ou seja, produtos produzidos no âmbito nacional com confiabilidade e rastreabi-

Comparações interlaboratoriais são a organização, desempenho e avaliação de ensaios nos mesmos itens ou em itens de ensaio similares, por dois ou mais laboratórios, de acordo com condições predeterminadas (ABNT ISO/IEC Guia 43-1, 1999).

\section{OBJETIVO}

A pouca disponibilidade de materiais de referência químicos no País constitui uma limitação que os laboratórios enfrentam no seu dia-a-dia. No Brasil, somente em junho de 2000, foi criada no Inmetro a Divisão de Metrologia Química (DQUIM), que tem a competência para: realizar ou reproduzir as unidades de medida; manter e conservar os padrões metrológicos nacionais que se acham sob sua responsabilidade; realizar o rastreamento dos padrões metrológicos nacionais aos internacionais por intermédio de comparações-chave, comparações internacionais e comparações regionais; colaborar e manter intercâmbio com o Bureau Internacional de Pesos e Medidas (BIPM) e outros organismos nacionais e internacionais, no âmbito da metrologia científica e industrial (INMETRO, 2003).

Para a Cgcre/Inmetro o problema é importante, pois dificulta o cumprimento dos critérios de acreditação pelos laboratórios.

O objetivo geral da pesquisa foi fazer um levantamento do uso de materiais de referência pelos laboratórios de ensaios em águas no Brasil, sob o ponto de vista da aquisição dos materiais, conforme o item "4.6 Aquisição de serviços e suprimentos” da ABNT NBR ISO/IEC 17025. Este artigo pretende investigar, com base na documentação que os laboratórios recebem dos fornecedores de materiais de referência, como é afetada a garantia da rastreabilidade das medições e, consequentemente, a qualidade dos serviços laboratoriais. lidade metrológica, adequados às normas e regulamentos técnicos internacionais, com a garantia da aplicação de procedimentos de avaliação da conformidade, visando minimizar custos com reensaios, recertificações, dentre outros (BORGES, 2007).

Segundo Hufbauer et al. (2000), os procedimentos de avaliação da conformidade de um determinado produto consistem em qualquer procedimento utilizado, direta ou indiretamente, para avaliar se certas exigências quanto a normas ou a regulamentos técnicos foram observadas. Além disso, tais procedimentos incluem amostragem, ensaios, inspeção, avaliação, verificação e garantia da conformidade, registro, acreditação e aprovação, bem como as combinações destas possibilidades. Os procedimentos de avaliação da conformidade são considerados essenciais em regulamentações relacionadas a saúde, segurança e meio ambiente e, contribuem para a melhoria da produtividade e a eficiência na economia de mercado (Apud, BORGES, 2007).

Em 2006, foi realizada uma pesquisa com 15 organismos de acreditação dos cinco continentes, representando $30 \%$ de todos os organismos de acreditação do Acordo da ILAC, visando a identificar, dentre outros, as principais não-conformidades impeditivas para realizar a avaliação nos laboratórios em fase de acreditação. A Tabela 1 apresenta o resultado da pesquisa, sendo pontos relevantes a não existência das políticas e dos procedimentos requeridos pela ISO/ IEC 17025 e procedimentos inadequados para calibração e para ensaios (SILVA, 2006).

O estudo relativo ao uso de materiais de referência no Brasil é relativamente recente. As "Diretrizes Estratégicas para a Metrologia Brasileira 2008-2012", que sucederam as diretrizes para o período 2003-2007, aprovaram várias metas relacionadas ao estabelecimento de referências metrológicas, dentre as quais o desenvolvimento de materiais de referência certificados. A Resolução do Conmetro, que 
aprovou as diretrizes, considera que a metrologia é uma área estratégica para o desenvolvimento econômico e social do País, por ser parte integrante da infra estrutura básica de apoio à competitividade das nossas empresas, à preservação da segurança, do meio ambiente e à proteção do consumidor. Ademais, o seu fortalecimento é uma forma de assegurar a soberania nacional neste importante segmento científico e tecnológico (CONMETRO, 2008).

Durante muito tempo, havia uma forma confusa de referir-se a materiais de referência, que por vezes era referido como "padrão", termo este que também era utilizado para designar artefatos materializados, especificações, métodos de ensaio e procedimentos de inspeção. Como consequência, o Conselho da ISO (órgão diretor da ISO) decidiu criar, em 1976, o Comitê sobre Materiais de Referência da ISO (Committee on Reference Materials - ISO REMCO) (PARKANY et al., 2001), visando a estudar e a promover o entendimento acerca dos materiais de referência.

O Comitê é responsável por elaborar e revisar os ISO Guides 30 a 35 que fornecem orientações sobre:

- termos e definições relacionados com materiais de referência;

- conteúdo de certificados e rótulos de materiais de referência);

- calibração em química analítica e uso de materiais de referência certificados;

- utilização de materiais de referência certificados;

- requisitos gerais para a competência de produtores de material de referência;

- princípios gerais e estatísticos para certificação de materiais de referência.

Em abril de 2005, o ISO REMCO aprovou novas definições para materiais de referência e materiais de referência certificados. Segundo Emmons et al. (2006) as definições da ISO Guide 30:1992 e do Vocabulário Internacional de Termos Fundamentais e Gerais da Metrologia (VIM) (2. ${ }^{\text {a }}$ edição) foram desenvolvidas na década de 1980 e foram baseadas nas necessidades e experiências das principais comunidades usuárias da época. Com o aumento da conscientização da garantia da qualidade analítica e o aumento dos organismos de normalização, de acreditação e dos reguladores que estão envolvidos nesse tema, foi preciso considerar outros aspectos na explicação desses termos para evitar confusão sobre conceitos.

As novas definições aprovadas são:

"Material de referência (MR)

Material suficientemente homogêneo e estável em relação a uma ou mais propriedades especificadas, o qual foi estabelecido como adequado ao uso pretendido em um processo de medição.

Nota 1: MR é um termo genérico.

Nota 2: As propriedades podem ser quantitativas ou qualitativas, por exemplo, identidade de substâncias ou espécies.

Nota 3: Os usos podem incluir a calibração de um sistema de medição, avaliação de um procedimento de medição, valores designados ou outros materiais, e controle da qualidade.

Nota 4: Um MR só pode ser usado para um único propósito em uma dada medição".

(EMMONS et al., 2006)

Emmons (2005) explica que a nota 1 da nova definição visa a esclarecer que o termo "material de referência" referese a uma família de materiais. Todos os materiais que tenham estabilidade e homogeneidade adequadas para controle da qualidade de uma medição pertencem à família do MR. Aqueles que não são acompanhados de um certificado são, em geral, denominados materiais de referência não certifica-

Tabela 1: Principais não-conformidades que impedem a realização da avaliação de acreditação.

\begin{tabular}{cc}
\hline Não-conformidades & $\%$ \\
\hline Procedimentos inadequados para calibração e para ensaio & 87,5 \\
Procedimentos inadequados para o cálculo de incerteza de medição & 87,5 \\
Falta de políticas requeridas & 75,0 \\
Falta de rastreabilidade & 75,0 \\
Falta de procedimentos requeridos & 75,0 \\
Políticas inadequadas & 62,5 \\
Procedimentos inadequados & 50,0 \\
Resultados insatisfatórios em comparações interlaboratoriais & 37,5 \\
\hline
\end{tabular}

Fonte: Silva, 2006 
dos. Mas também são utilizados muitos outros termos como materiais produzidos internamente, materiais de controle do laboratório, materiais de referência do laboratório.

"Material de referência certificado (MRC)

Material de referência, caracterizado por um procedimento metrologicamente válido, para uma ou mais propriedades, acompanhado de um certificado que fornece o valor da propriedade especificada, sua incerteza associada e uma declaração da rastreabilidade metrológica."

(EMMONS et al., 2006)

Convém mencionar que o ABNT ISO Guia 31:2004 resume o conteúdo essencial de um certificado de MRC: nome do material; produtor e código do produtor para o material; descrição geral do material; uso pretendido; instruções para o uso apropriado; instruções para as condições de armazenamento; valor(es) de propriedade certificado(s), cada um deles acompanhado de uma declaração de incerteza; método(s) empregado(s) para a obtenção de valores de propriedade (com detalhes completos quando os valores forem dependentes do método de medição); e, prazo de validade, se apropriado.

A norma ABNT NBR ISO/IEC 17025, utilizada na acreditação de laboratórios, contém requisitos para competência de laboratórios, escritos sob a perspectiva das boas práticas de gestão e de técnicas de um laboratório. Alguns destes requisitos tratam da seleção e do uso de materiais de referência. São eles:

- Requisito da direção - Análise crítica de pedidos, propostas e contratos (item 4.4). O laboratório deve ter capacidade e recursos (inclusive os materiais de referência) para atender aos requisitos dos clientes.

- Requisito da direção - Aquisição de serviços e suprimentos em laboratório de ensaio (item 4.6). Esse item referese a políticas e procedimentos, verificação, documentos de compra e fornecedores aprovados. Todos os serviços e suprimentos tratados nesse item são aqueles que afetam a qualidade dos ensaios e/ou calibrações. O tipo de serviço mais comum ao qual se refere o requisito é a calibração de equipamentos (A2LA, 2006). Este serviço interfere na qualidade dos resultados de ensaios, pois está diretamente relacionado à manutenção da rastreabilidade das medições. Pelo mesmo motivo, quando são adquiridos materiais de referência num laboratório de ensaios químicos, deve-se ter o cuidado de selecionar aqueles adequados ao uso pretendido. Uma vez assegurada a rastreabilidade metrológica, o requisito 5.6 da ABNT NBR ISO/IEC 17025 fica válido.

- Requisito técnico - Métodos de ensaio e calibração e validação de métodos (item 5.4). No aspecto geral, a norma estabelece que o laboratório deva utilizar métodos e procedimentos apropriados para todos os ensaios e/ou calibrações dentro do seu escopo de trabalho, deve ter todas as instruções necessárias, atualizadas e disponíveis ao seu pessoal, e garantir que desvios aos métodos sejam documentados, tecnicamente justificados, autorizados e aceitos pelo cliente. Utilizar "métodos e procedimentos apropriados" significa que estes devem ser adequados para um determinado propósito ou fim, que já foi previamente acordado com o cliente, conforme visto acima na análise crítica de contrato.

- A metodologia de ensaio de águas da natureza mais difundida é a do Standard Methods for the Examination of Water and Wastewater (SMEWW) e para quase todos os parâmetros mencionados existe um método correspondente.

- Requisito técnico - Rastreabilidade de medição (item 5.6). Para laboratório de ensaios, deve haver um programa de calibração para os equipamentos de medição e ensaio utilizados com funções de medição, operados de forma a assegurar que as medições feitas pelo laboratório sejam rastreáveis ao Sistema Internacional (SI). Nos casos em que a rastreabilidade das medições às unidades SI não for possível e/ou não for pertinente, a norma dispõe que se deve utilizar materiais de referência certificados e métodos e/ou padrões de consenso (todas as partes envolvidas) como referências para obter-se rastreabilidade.

O Vocabulário Internacional de Termos Fundamentais e Gerais de Metrologia (VIM, 2007, item 2.41) define rastreabilidade metrológica como:

"Propriedade de um resultado de medição pela qual tal resultado pode ser relacionado a uma referência através de uma cadeia ininterrupta e documentada de calibrações, cada uma contribuindo para a incerteza de medição".

Define também incerteza de medição (VIM, 2007, item 2.26) como:

"Parâmetro não negativo que caracteriza a dispersão dos valores atribuídos a um mensurando, com base nas informações utilizadas".

Segundo a definição, a rastreabilidade é uma propriedade do resultado de medição e, portanto a frase muito comum "rastreável à instituição X" é uma simplificação de "rastreável a um valor de referência mantido pela instituição X". Da mesma forma, "rastreável ao SI" é uma simplificação de "rastreável ao valor de referência obtido pela realização das unidades acordadas do SI" (ELLISON et al., 2003). 
No caso do Brasil, a Cgcre/Inmetro estabeleceu a política de rastreabilidade ao SI de unidades para garantir que os laboratórios brasileiros efetuem a calibração de seus padrões de referência e instrumentos em laboratórios que possam demonstrar competência, capacidade de medição e rastreabilidade para a calibração específica a ser executada (INMETRO, 2003).

Requisito técnico - Garantia da qualidade dos resultados de ensaio e calibração (item 5.9). O requisito estabelece que sejam implantadas medidas de controle da qualidade para o monitoramento da validade dos ensaios e calibrações. São sugeridas algumas técnicas, incluindo o uso regular de materiais de referência certificados e/ou controle interno da qualidade com materiais de referência secundários. Convém que as técnicas utilizadas sejam apropriadas para o tipo e volume do trabalho realizado.

Mas como encontrar o material de referência apropriado? Conforme afirma Steiger (2006), constantemente os usuários de materiais de referência se deparam com a dificuldade de encontrar o material apropriado: "Existe um material de referência disponível que atenda aos requisitos analíticos (analitos, matriz, nível de concentração, incertezas)? O produtor do material de referência é confiável?’. Por isso, foi desenvolvida a base internacional de dados sobre materiais de referência certificados COMAR (Code d'Indexation des Matériaux de Référence), via "web", de acesso livre pela Internet no endereço <www.comar.bam.de>. Existem outras bases de dados como a da International Atomic Energy Agency (IAEA), a CRM Producers - Worldwide listing of CRM producers, além de algumas administradas por organizações regionais, mas somente a COMAR cobre uma gama de materiais de referência certificados de produtores de vários países. Atualmente a COMAR congrega 19 centros de codificação, situados em países da Europa, América do Norte, Ásia e Oceania. Os materiais de referência certificados catalogados devem atender às recomendações dos ISO Guides 31, 34 e 35. Os campos de aplicação definidos para cadastro na COMAR são: ferrosos, não ferrosos, inorgânicos, orgânicos, propriedades físicas, biológicos, qualidade de vida e indústria (COMAR, 2008).

Consultando o banco de informações da COMAR sobre materiais de referência certificados brasileiros, verificou-se que somente uma instituição brasileira consta nessa base de dados, o Instituto de Pesquisas Tecnológicas do Estado de S.Paulo (IPT), cujos materiais não se aplicam aos ensaios de águas. Ocorre que, além da dificuldade de encontrar a informação, os materiais de referência certificados importados são caros e de difícil obtenção, devido aos trâmites burocráticos impostos pelas autoridades alfandegárias, não só do Brasil, mas também dos demais países do mundo. Sobre essa questão, o ISO REMCO está dando andamento a um estudo para conhecer os problemas relacionados ao transporte, importação e exportação e barreiras ao comércio de materiais de referência.

\section{A Agência Nacional de Águas (ANA) e a Cgcre/Inmetro rede de laboratórios acreditados para análise de águas.}

Conforme já mencionado, o PROLAB estabeleceu critérios para um laboratório de análise de água participar do Programa. Dentre as exigências estão que os laboratórios devem seguir os requisitos da ABNT NBR ISO/IEC 17025 e os parâmetros a serem analisados e respectivos métodos analíticos devem estar em conformidade com as normas técnicas brasileiras e/ou do Mercosul ou, na ausência destas, normas técnicas internacionais cientificamente reconhecidas.

\section{METODOLOGIA DE PESQUISA}

A pesquisa que gerou este artigo é classificada como exploratória, pois "é realizada em área na qual há pouco conhecimento acumulado e sistematizado". A hipótese para a realização desta investigação é que os laboratórios nacionais de análise de águas não utilizam os materiais de referência, preconizados pela norma ABNT NBR ISO/IEC 17025, para garantir a rastreabilidade metrológica dos resultados dos ensaios executados; também se enquadra como aplicada porque é motivada pela necessidade de resolver problemas concretos (VERGARA, 2005, p. 47), considerando que os resultados da pesquisa têm como objetivo subsidiar o organismo de acreditação na elaboração de uma política a ser seguida pelos laboratórios para a questão aqui apresentada.

Do ponto-de-vista da forma de abordagem, pode ser considerada uma pesquisa qualitativa. Para Neves (1996, p. 1), enquanto os estudos quantitativos geralmente procuram seguir com rigor um plano previamente estabelecido, a pesquisa qualitativa costuma ser direcionada ao longo de seu desenvolvimento. Além disso, não busca enumerar ou medir eventos e, geralmente, não emprega ferramentas estatísticas para a análise dos dados - seu foco de interesse é amplo e parte de uma perspectiva diferente daquela adotada pela pesquisa quantitativa. 
É, ainda, um estudo de caso, pois foi pesquisado apenas o universo de laboratórios de análises de água, não considerando os laboratórios voltados para outros tipos de ensaios.

Na execução do estudo foi utilizado um questionário (estudo de campo), elaborado em planilha Excel, e enviado aos laboratórios pertencentes ao banco de dados de Laboratórios de Ensaios e de Calibração Acreditados (RBC/RBLE) disponível na homepage do Inmetro, por correio eletrônico. Segundo Marconi e Lakatos (2008, p. 86) questionário é um instrumento de coleta de dados constituído por uma série ordenada de perguntas que devem ser respondidas por escrito e sem a presença do entrevistador. Como técnica de coleta de dados, o questionário apresenta uma série de vantagens e desvantagens. Algumas vantagens são: economiza tempo, viagens e obtém grande número de dados; abrange uma área geográfica mais ampla; obtém respostas mais rápidas e mais precisas; há menos risco de distorção pela não influência do pesquisador. Algumas desvantagens podem ser: percentagem pequena de questionários que voltam; impossibilidade de ajudar o informante em questões mal compreendidas. O envio de questionários por meio eletrônico deveu-se à necessidade de incluir todos os laboratórios do universo da pesquisa, distribuídos por vários Estados do País, o que dificultaria a entrevista pessoal. Primeiro foi perguntado aos laboratórios que materiais de referência (MR) são utilizados como padrão nas análises/ensaios dos parâmetros de interesse. A cada laboratório foi solicitado que respondesse somente uma opção - aquela que fosse a mais freqüente - dentre as 11 respostas possíveis. Depois foi pedido aos laboratórios que indicassem que tipo de certificação ou acreditação, por uma organização independente (de terceira parte), é encontrado no certificado do material ou no certificado de análise que acompanha o material de referência. A cada laboratório foi solicitado que respondesse somente uma opção - aquela que fosse a mais frequente - dentre as quatro respostas possíveis. Neste arti- go serão discutidas as respostas sobre esta última pergunta, mostrando resultados obtidos para os parâmetros.

O universo da pesquisa de campo compreendeu todos os laboratórios de ensaios de águas que prestam serviços analíticos para a ANA: os acreditados, os em fase de acreditação pela Cgcre/Inmetro, e outros laboratórios não acreditados que também prestam serviços à ANA e que participaram de uma comparação interlaboratorial organizada pela Diretoria de Metrologia Científica e Industrial (Dimci) do Inmetro em parceria com a Cgcre/Inmetro.

\section{RESULTADOS E DISCUSSÃO}

O questionário da pesquisa de campo apresentou um quadro quantitativo de respostas mostrado na Tabela 2. Segundo Marconi e Lakatos (2008, p. 86), em média, os questionários expedidos alcançam $25 \%$ de devolução. São fatores que exercem influência no retorno dos questionários: "o patrocinador, a forma atraente, a extensão, o tipo de carta que o acompanha, solicitando colaboração; as facilidades para seu preenchimento e sua devolução pelo correio (C. SELLTIZ, 1965, apud MARCONI; LAKATOS, 2008). No total, foram consultados os 62 laboratórios de análises de água prestadores de serviços analíticos para a ANA (que compõem o universo da pesquisa), sobre cada um dos 53 parâmetros. O total de respondentes foi de 31 , sendo que 17 destes são laboratórios acreditados. Ou seja, em relação ao número de questionários respondidos, mais de $50 \%$ foram os laboratórios acreditados, que evidenciaram a implantação dos requisitos da ABNT NBR ISO/IEC 17025. A quantidade de respostas maior do que a média esperada (25\%) pode se dever ao fato das facilidades existentes no relacionamento via correio eletrônico. O questionário enviado foi submetido a um pré-teste para verificação de possíveis falhas.

Tabela 2: Questionários enviados x respondidos, de acordo com o status da acreditação.

\begin{tabular}{|c|c|c|c|}
\hline \multirow[t]{2}{*}{ Status da acreditação do laboratório } & \multicolumn{2}{|c|}{$\%$ de funcionários } & \multirow{2}{*}{$\begin{array}{l}\% \text { de funcionários respondidos por } \\
\text { status da acreditação, em relação } \\
\text { ao } n^{\circ} \text { total de respondidos }\end{array}$} \\
\hline & enviados & respondidos & \\
\hline acreditado & 23 & 17 & $54,8 \%$ \\
\hline em fase de acreditação & 28 & 09 & $29,0 \%$ \\
\hline $\begin{array}{l}\text { participantes da comparação } \\
\text { interlaboratorial, não acreditados ou } \\
\text { em fase de acreditação }\end{array}$ & 11 & 05 & $16,2 \%$ \\
\hline Total & 62 & 31 & 50,0 \\
\hline
\end{tabular}


Dentre os 53 parâmetros do PROLAB, serão discutidos neste trabalho resultados dos cinco parâmetros considerados básicos pelo Programa, e um grupo de metais pesados na matriz água, tóxicos ao homem e ao meio ambiente. Ao final, são apresentadas as respostas obtidas para todos os parâmetros, mostrando uma visão geral do tipo de certificação ou de acreditação por uma organização independente (de terceira parte) encontrada no certificado do material ou no certificado de análise que acompanha o material de referência. Cabe também esclarecer que os resultados da pesquisa com valores menores do que $10,0 \%$ como resposta não foram comentados.

O sistema de informações hidrológicas da Agência Nacional de Águas desenvolve uma série de ações como, por exemplo, avaliação ambiental por bacia hidrográfica; avaliação da rede hidrográfica existente incluindo a qualidade da água; adequação da rede de qualidade da água existente e dos parâmetros analisados em função dos eventos potencialmente impactantes. Sendo assim, convém dar atenção a alguns parâmetros da qualidade da água, especialmente aqueles passíveis de se medir em campo, e que sugerem diferentes tipos de poluição. Dentre esses parâmetros destacam-se: $\mathrm{pH}$, associado à poluição de origem industrial e à qualidade das águas para consumo humano e industrial e para manutenção das comunidades aquáticas; o oxigênio dissolvido (OD), associado à poluição tanto industrial quanto doméstica; a condutividade elétrica, associada à qualidade das águas para irrigação e para uso industrial; a turbidez, associada ao conteúdo de sólidos suspensos na água e aos processos erosivos da bacia contribuinte (PROLAB, 2005).

Como informado anteriormente, consta do banco de informações da COMAR somente uma instituição brasileira, o Instituto de Pesquisas Tecnológicas do Estado de S.Paulo (IPT), cujos materiais não se aplicam aos ensaios de águas. Convém lembrar mais uma vez que os materiais de referência certificados importados são caros e de difícil obtenção.

\section{$5.1 \mathrm{pH}$, oxigênio dissolvido, condutividade, temperatura e turbidez}

Para a avaliação do $\mathrm{pH}$, o Standard Methods recomenda, no método $4500 \mathrm{H}^{+} \mathrm{B}$ (método eletrométrico), o uso de solução tampão do NIST, que no Brasil seria correspondente a uma solução tampão produzida pelo Inmetro.

A maioria dos certificados recebidos pelos laboratórios $(46,7 \%)$ não faz nenhum tipo de referência a reconhecimento por terceira parte, o que pode ser observado na Tabela
3. Um percentual menor dos laboratórios $(26,7 \%)$ relata encontrar referência à certificação ISO 9001- requisitos para sistemas de gestão da qualidade- que não está relacionada aos requisitos de competência técnica do ABNT ISO Guia 34 ou da ABNT NBR ISO/IEC 17025.

Para o oxigênio dissolvido (OD), o Standard Methods recomenda utilizar os métodos iodométrico e o eletrométrico, os quais incluem etapa de padronização de solução para titulação. Os 41,9\% que responderam não haver referência a reconhecimento por terceira parte incluem os laboratórios que utilizam reagente pró-analisis (PA) e os que não utilizam padrão na confecção da solução para titulação (Tabela 3).

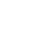

\section{a implementação dos requisitos para "Aquisição de serviços e suprimentos" da ABNT NBR ISO/IEC 17025.}

Para a condutividade elétrica, o Standard Methods recomenda, no procedimento $2510 \mathrm{~B}$ (medição em laboratório condutivímetro), o preparo de uma solução padrão de referência.

A maioria dos certificados recebidos pelos laboratórios $(45,2 \%)$ não faz nenhum tipo de referência a reconhecimento por terceira parte. Um percentual menor $(22,6 \%)$, apresentado na Tabela 3, relata encontrar referência à certificação ISO 9001 que não está relacionada aos requisitos de competência técnica do ABNT ISO Guia 34 ou da ABNT NBR ISO/IEC 17025.

A turbidez é uma expressão da propriedade ótica que faz com que a luz seja dispersa e absorvida, ao invés de ser transmitida sem alteração na sua direção ou no nível do fluxo através da amostra. O Standard Methods recomenda, no procedimento $2130 \mathrm{~B}$ (método nefelométrico), o uso de uma suspensão padrão de referência, que pode ser preparada no laboratório. Os resultados desse ensaio são expressos em NTU (unidades de turbidez nefelométrica). A maioria dos certificados recebidos pelos laboratórios $(41,9 \%)$ não faz nenhum tipo de referência a reconhecimento por terceira parte. Um percentual menor $(19,5 \%)$ relatou encontrar referência à certificação ISO 9001 (ver Tabela 3).

\subsection{Metais (cádmio, chumbo, cobalto, cobre, níquel, prata e zinco)}

Os efeitos dos metais em água e efluentes podem ser variados, benéficos, prejudiciais ou extremamente tóxicos. 
Alguns metais são essenciais ao crescimento dos animais e dos vegetais, enquanto outros podem afetar adversamente os consumidores de água e os sistemas de tratamento de efluentes. Os benefícios e toxidez dependem das suas concentrações em água.

O Standard Methods prescreve vários procedimentos para determinação de metais, por espectrometria de absorção atômica, espectrometria de emissão de plasma, método eletrométrico para chumbo, cádmio e zinco, método colorimétrico para chumbo, métodos da neocuproína e da batocuproína. Todos os procedimentos requerem a construção de curva de calibração.

A maioria dos certificados recebidos pelos laboratórios $(35,5 \%)$ não contém referência a reconhecimento por terceira parte. Um percentual menor, 19,4\%, relata encontrar referência à certificação ISO 9001 que não está relacionada aos requisitos de competência técnica do ABNT ISO Guia 34 ou da ABNT NBR ISO/IEC 17025 (ver Tabela 4).

\subsection{Arsênio total}

O arsênio é usado em ligas com chumbo, em baterias e munições. Os compostos de arsênio são muito utilizados em pesticidas e na preservação de madeiras. $\mathrm{O}$ arsênio não é elemento essencial às plantas, mas, em traços, é essencial a várias espécies animais. A toxidez do arsênio depende da forma química. Alguns compostos de arsênio são considerados cancerígenos.

O Standard Methods recomenda, além dos procedimentos espectrométricos utilizados para metais, o método do ditiocarbamato de prata. Todos os procedimentos requerem construção de curvas de calibração.

Os resultados desta pesquisa para o parâmetro "arsênio total" foram similares aos resultados de metais discutidos em 5.2, conforme pode ser observado na Tabela 4.

\subsection{Bário total}

O bário é usado nas lamas de perfuração de poços de petróleo, venenos de ratos, pigmentos, produtos pirotécnicos e em medicamentos. Dependendo da concentração, o bário é tóxico ao ambiente marinho.

O Standard Methods recomenda, para determinação de bário, os procedimentos espectrométricos utilizados para metais. Esses procedimentos requerem a construção de curvas de calibração.

Os resultados desta pesquisa para o parâmetro "bário total" também foram similares aos resultados de metais discutidos em 5.2, conforme pode ser observado na Tabela 4 .

Tabela 3: Certificados de MR ou de análise com acreditação/certificação.

\begin{tabular}{ccccc}
\hline Respostas & $\mathrm{pH}$ & $\mathrm{OD}$ & Condutividade & Turbidez \\
& $(\%)$ & $(\%)$ & $25)$ & 25,8 \\
\hline Não analisam este parâmetro & 16,7 & 35,5 & 22,6 & 9,7 \\
Não responderam & 3,3 & 16,1 & 3,2 & 19,5 \\
Referência à certificação ISO 9001 & 26,7 & 3,2 & 22,6 & --- \\
Acreditação ISO/IEC 17025 & 3,3 & ---- & 3,2 & 3,2 \\
Produtor de MR conforme ISO Guide 34 & 3,3 & 3,2 & 45,2 & 41,9 \\
Nenhuma dessas referências & 46,7 & 41,9 & & \\
\hline
\end{tabular}

Tabela 4: Metais, arsênio total e bário total - Certificados de MR ou de análise com acreditação/ certificação.

\begin{tabular}{cccc}
\hline Respostas & Metais & As & Ba \\
$(\%)$ & $(\%)$ & 45,2 \\
Não analisam este parâmetro & 41,9 & 41,9 & ---- \\
Não responderam & ---- & ---- & 12,9 \\
Referência à certificação ISO 9001 & 19,4 & 12,9 & --- \\
Referência à acreditação ISO/IEC 17025 & ---- & --- & 3,2 \\
Referência à acreditação do produtor de MR conforme ISO Guide 34 & 3,2 & 3,2 & 38,7 \\
Nenhuma dessas referências & 35,5 & 41,9 & 3 \\
\hline
\end{tabular}


Na Tabela 5, é apresentada uma consolidação das respostas para todos os 53 parâmetros $(\mathrm{pH}$, condutividade, oxigênio dissolvido, turbidez, cor, teor de sólidos, parâmetros biológicos, indicadores de matéria orgânica, metais, agrotóxicos etc.). A maioria dos certificados recebidos pelos laboratórios de água pesquisados não contém referência alguma a reconhecimento por terceira parte. Uma proporção bem menor dos laboratórios relata encontrar referência à certificação ISO 9001 que não está relacionada aos requisitos de competência técnica do ABNT ISO Guia 34 ou da ABNT NBR ISO/IEC 17025. No entanto, no grupo dos laboratórios postulantes à acreditação, a maior incidência é de certificados com referência à norma ISO 9001.

Uma proporção pequena afirmou encontrar referência a reconhecimento de terceira parte baseada na norma $\mathrm{ABNT}$ NBR ISO/IEC 17025 ou no ISO Guide 34.

\subsection{Proposta de melhoria da rastreabilidade}

Considerando que diversos fatores determinam a correção e confiabilidade dos ensaios realizados, inclusive fatores humanos, acomodações e condições ambientais, métodos de ensaio e validação de métodos, equipamentos, amostragem, manuseio de itens de ensaio e rastreabilidade da medição (ABNT NBR ISO/IEC 17025, item 5.1.1), uma proposta de melhoria pode ser considerada, baseada em Engelhard et al. (2004) e nos resultados obtidos com esta pesquisa realizada e que foi apresentada ao Inmetro visando a garantir a rastreabilidade dos resultados de ensaios dos laboratórios, quando há dificuldade em obter materiais de referência certificados. Os pontos dessa proposta são:

- o laboratório de ensaio deve calibrar os seus equipamentos em laboratório com competência demonstrada para o serviço de calibração pertinente. O laboratório de ensaio deve seguir a política de participação em atividade de ensaio de proficiência estabelecida no documento da Cgcre/Inmetro NIT-DICLA-026, de forma a demonstrar comparabilidade dos seus resultados de ensaio com os seus pares;

- o laboratório de ensaio deve procurar por MR rastreáveis, preferencialmente MRC; deve demonstrar que buscou na base de materiais de referência COMAR e que segue as orientações do DOQ-CGCRE 016 para seleção e uso de materiais de referência;

- o laboratório de ensaio deve questionar o produtor do material de referência se tem ou pretende pedir acreditação segundo a ABNT NBR ISO/IEC 17025, ou ISO Guide 34, pois um MR de um produtor acreditado reduz custos para o laboratório e facilita a confirmação da rastreabilidade. O certificado do material de referência deve estar de acordo com o ABNT ISO Guia 31, demonstrando que o laboratório aplica o item 4.6 da ABNT NBR ISO/IEC 17025;

- o laboratório de ensaio deve trabalhar com um método normalizado. Quando este não existir, o laboratório deve proceder à validação do método, seguindo as orientações do DOQ-CGCRE-008;

- o laboratório de ensaio deve definir as incertezas dos resultados e as variáveis do método de ensaio que podem afetar a qualidade do resultado do ensaio. Cuidar para que essas variáveis sejam rastreáveis às unidades SI, sempre que possível, seguindo as orientações do EURACHEM/ CITAC Guide Quantifying Uncertainty in Analytical Measurement. O laboratório de ensaio deve proceder ao controle da qualidade na implantação do método no laboratório e na sua aplicação na rotina (curva de calibração x resposta, cartas de controle etc.);

- o laboratório de ensaio deve proceder às verificações intermediárias de balanças, termômetros etc.

Tabela 5: Certificados de MR ou de análise com acreditação/certificação - Resultados consolidados para os 53 parâmetros analisados.

\begin{tabular}{ccccc}
\hline Respostas & $\begin{array}{c}\text { Todos } \\
\%\end{array}$ & $\begin{array}{c}\text { Acreditados } \\
\%\end{array}$ & $\begin{array}{c}\text { Postulantes } \\
\%\end{array}$ & $\begin{array}{c}\text { Participantes do } \\
\text { Interlaboratorial } \\
\%\end{array}$ \\
\hline $\begin{array}{c}\text { Não analisam este parâmetro (pH, condutividade, OD, } \\
\text { turbidez, metais e os demais parâmetros do PROLAB) }\end{array}$ & 49,7 & 44,4 & 59,3 & 50,2 \\
Não responderam & 5,2 & 3,2 & 7,1 & 22,2 \\
Referência à certificação ISO 9001 & 12,7 & 8,8 & 3,6 & 9,0 \\
Acreditação ISO/IEC 17025 & 1,5 & 0,8 & 1,0 & 0,0 \\
Produtor de MR conforme ISO Guide 34 & 1,5 & 0,2 & 6,0 & 6,8 \\
Nenhuma dessas referências & 29,4 & 42,6 & 25,7 & 6,7 \\
\hline
\end{tabular}


- o laboratório de ensaio deve preparar soluções em frascos calibrados e proceder a verificações intermediárias com soluções de trabalho;

- o laboratório de ensaio deve documentar e monitorar dados históricos coletados para identificação de tendências;

- quando do preparo de MR de trabalho no laboratório, este deve assegurar-se de que o equipamento esteja calibrado, deve monitorar a estabilidade e homogeneidade do MR, documentar procedimentos e os resultados obtidos. Quando não houver MRC de um produtor de MR acreditado ou de um instituto nacional de metrologia, o laboratório de ensaio deve: verificar se o certificado de análise recebido com o composto define o tipo de MR; e solicitar informação adicional do fornecedor do material sobre estabilidade, homogeneidade, rastreabilidade e incerteza para os valores declarados no certificado de análise, aplicando o requisito 4.6 da ABNT NBR ISO/IEC 17025.

- O laboratório de ensaio deve manter documentadas todas as informações disponíveis sobre o MR (coletar dados e manter histórico), manter conhecimento histórico sobre o fornecedor de MR, visando o controle da qualidade dos ensaios e propiciando o conhecimento sobre o processo de medição.

- Nos casos em que nada do que foi recomendado acima puder ser executado, usar o método de ensaio sempre do mesmo modo, com os mesmos materiais e nas mesmas condições, com controles apropriados (particularmente para métodos empíricos). Fixar todas as variáveis do processo de medição, como analista, equipamento, amostra, método, visando controlar as fontes de erro no processo.

Todas as proposições acima só podem ser implementadas com sucesso com pessoal de laboratório adequadamente treinado, o que não é tarefa simples, conforme ilustra uma pesquisa da ILAC, feita em 2004, junto a organismos de acreditação de 51 países, para verificar os problemas encontrados pelos laboratórios em relação à conversão do antigo ISO Guide 25 para a primeira versão da ISO/IEC 17025 em 1999. O resultado mostrou que, na visão dos gerentes dos laboratórios, ainda havia carência de treinamento em temas como estimativa de incerteza de medição, validação de métodos, comparações interlaboratoriais, rastreabilidade e tópicos relacionados ao sistema de gestão da qualidade (McNAIR, 2004).

O detalhamento para implantação desta política pode ser encontrado no trabalho de Moura (2006, p.190).

Conforme constatado nesta pesquisa, a utilização de poucos certificados de MR com reconhecimento de ter- ceira parte, de acordo com a ISO/IEC 17025 ou ISO Guide 34 - normas que tratam efetivamente da rastreabilidade - poderá acarretar em barreiras técnicas e poderá trazer sérios problemas na análise das águas, como apontado pela ANA.

Uma das causas de os certificados não apresentarem reconhecimento de terceira parte pode estar relacionada ao fato de o usuário não exigir do produtor o fornecimento de certificados de análise reconhecidos. O usuário (laboratório), por sua vez, também pode não estar sendo exigido pelo organismo de acreditação, Cgcre/Inmetro. O cliente do laboratório, que também é parte interessada no sistema de acreditação de laboratórios, também não é exigente nesse aspecto. A infraestrutura para tratar de materiais de referência (e sua rastreabilidade) no País ainda não é capaz de atender às necessidades dos laboratórios. Sabe-se que, por enquanto, o suprimento das necessidades por materiais de referência na maioria dos países do mundo é insuficiente. Com a ILAC sinalizando um futuro acordo de reconhecimento mútuo para produção de materiais de referência, a Cgcre/Inmetro deve preparar-se para tal, sob o risco de ocorrer uma real defasagem tecnológica.

Apesar dos esforços de se implantar a metrologia química no País, ficou evidente que ainda há carência de materiais de referência que atendam aos laboratórios de análise de águas. Desse modo, a implementação do item "Aquisição de serviços e suprimentos" (4.6) da ABNT NBR ISO/IEC 17025 por parte do laboratório de ensaio, com políticas e procedimentos para a seleção e compra de serviços e suprimentos utilizados que afetem a qualidade dos ensaios, irá modificar as relações cliente/fornecedor promovendo uma melhoria da qualidade no fornecimento de materiais de referência.

\section{CONCLUSÕES}

Como estratégia no curto prazo, este trabalho foi apresentado à Cgcre/Inmetro, indicando a proposta de uma política para os laboratórios de ensaios integrantes do seu sistema de acreditação, conforme Capítulo 5 deste artigo, com vistas a efetivar a implementação dos requisitos para "Aquisição de serviços e suprimentos" da ABNT NBR ISO/IEC 17025 por parte dos laboratórios, caracterizando os estudos práticos de implantação das etapas da política proposta nesta pesquisa.

Como estratégia no médio prazo, a Cgcre/Inmetro está implementando um programa de acreditação de produtores de materiais de referência, em consonância com normas internacionais, além do programa de acreditação de provedores de ensaios de proficiência para apoiar a política de 
participação em atividades de ensaios de proficiência estabelecida na NIT-DICLA-026.

Finalmente, os resultados e recomendações constantes deste trabalho pretendem alertar a sociedade civil, os laboratórios, os centros de pesquisa, acadêmicos ou não, e os governos para a importância do tema, considerando que conclusões equivocadas quanto ao resultado de análises sejam de água ou de outras substâncias que possam comprometer a saúde dos seres vivos e a sobrevivência do ecossistema, podem ser irreversíveis e, portanto, de grande importância para garantir a sustentabilidade atualmente tão buscada.

\section{Artigo recebido em 27/06/2007 Aprovado para publicação em 26/03/2009}

\section{REFERÊNCIAS}

AGÊNCIA NACIONAL DE ÁGUAS - ANA. $A$ água no Brasil e no Mundo. Disponível em: http://www.ana.gov.br. Acesso em: 18 jul. 2005.

Programa Nacional de Acreditação de Laboratórios em Análises da Qualidade da Água - PROLAB - Documento Base. Maio de 2005. Disponível em http://www.ana.gov.br. Acesso em: 24 ago. 2005.

AMERICAN ASSOCIATION FOR LABORATORY ACCREDITATION - A2LA. Understanding ISO/ IEC 17025: A2LA Specific applications of the standard. 27 fev. 2006. p. 6/16. Disponível em: http://www.a2la.org. Acesso em: 28 fev. 2006

AMERICAN PUBLIC HEALTH ASSOCIATION; AMERICAN WATER WORKS ASSOCIATION: WATER ENVIRONMENT FEDERATION. Standard Methods for the Examination of Water and Wastewater. 21st Edition, 2005.

ASSOCIAÇÃO BRASILEIRA DE NORMAS TÉCNICAS - ABNT. ABNT NBR ISO/IEC 17000: Avaliação da conformidade - Vocabulário e princípios gerais. Rio de Janeiro, 2005. 18 p.

ABNT NBR ISO/IEC 17025: Requisitos gerais para a competência de laboratórios de ensaio e calibração. Rio de Janeiro, 2005. 31 p.

ABNT ISO Guia 31: Materiais de referência - Conteúdo de certificados e rótulos. Rio de Janeiro, 2004. 8 p.
. ABNTISO Guia 32: Calibração em química analítica e uso de materiais de referência certificados. Rio de Janeiro, 2000. 7 p.

ABNT ISO Guia 33: Utilização de materiais de referência certificados. Rio de Janeiro, 2002. 28 p.

ABNT ISO Guia 34: Requisitos gerais para a competência de produtores de material de referência. Rio de Janeiro, 2004. 25 p.

ABNT NBR ISO/IEC Guia 43-7: Ensaios de proficiência por comparações interlaboratoriais - Parte 1: Desenvolvimento e operação de programas de ensaios de proficiência. Rio de Janeiro, 1999. 17p.

ABNT NBR ISO 9001: Sistemas de gestão da qualidade - Requisitos. 2. ed. Rio de Janeiro, 2008. 28 p.

BORGES, R. M. H. A metrologia como um instrumento aos laboratórios: o caso de laboratórios de análises de combustíveis. Tese de Doutorado - Universidade Federal do Rio de Janeiro, Escola de Química, 2007.

BUREAU INTERNATIONAL DE POIDS ET MESURES - BIPM. JCGM 200:2008. International vocabulary of metrology - Basic and general concepts and associated terms (VIM). 3. ed. Disponível em: http://www.bipm.org. Acesso em 5 dez. 2008.
BRASIL. Conselho Nacional do Meio Ambiente. Resolução n. 357, de 17 de março de 2005 Disponível em: http://www.ana.gov.br. Acesso em: 11 jul. 2005.

COMAR, THE INTERNATIONAL DATABASE FOR CERTIFIED REFERENCE MATERIALS (CRM). Disponível em: http://www.comar.bam. de. Acesso em: 5 dez. 2008.

CONSELHO NACIONAL DE METROLOGIA NORMALIZAÇÃO E QUALIDADE INDUSTRIAL - CONMETRO. Resolução n. 8, de 16 de setembro de 2008. Dispõe sobre a aprovação do documento Diretrizes Estratégicas para a Metrologia Brasileira 2008-2012. Disponível em http://www.inmetro.gov.br. Acesso em: 7 nov. 2008.

ELLISON, S. L. R.; KING, B.; RÖSSLEIN, M.; SALIT, M.; WILLIAMS, A. (Drafting editors). EURACHEM/CITAC Guide: traceability in chemical measurement - $A$ guide to achieving comparable results in chemical measurement. 2003. Disponível em: http://www.eurachem. org. Acesso em: 5 dez. 2008.

EURACHEM/CITAC Guide Quantifying Uncertainty in Analytical Measurement. 2. ed., 2000. Disponível em: http://www.eurachem. org. Acesso em: 5 dez. 2008.

EMMONS, H.; FAJGELJ, A.; VAN DER VEEN, A.; WATTERS, R. New definitions on reference materials. Accreditation and quality assurance. Springer Verlag, 2006. DOI.1007/s00769-0060089-9. 
EMMONS, Hendrik. The "RM family" Identification of all of its members. Accreditation and quality assurance. Springer Verlag, 2005. DOI 10.1007/s00769- 005-0046-z.

ENGELHARD, T.; DREAZEN, O. Current best practice for traceability in testing laboratories, when certified reference materials are unavailable. Accreditation and quality assurance. Springer Verlag, v. 9, p. 397-398, 2004.

INSTITUTO NACIONAL DE METROLOGIA, NORMALIZAÇÃO E QUALIDADE INDUSTRIAL - Inmetro. Vocabulário internacional de termos fundamentais e gerais da metrologia - VIM. Duque de Caxias, 1995. 52 p.

NIT-DICLA-026: Requisitos sobre a participação dos laboratórios de ensaios e de calibração em atividades de ensaios de proficiência. Revisão 03, dezembro de 2005. Disponível em: http://www.inmetro.gov.br. Acesso em: 18 fev. 2006.

NIT-DICLA-030: Rastreabilidade ao sistema internacional de unidades no credenciamento de laboratórios. Revisão 01, julho de 2003. Disponível em: http://www.inmetro.gov. br. Acesso em: 18 fev. 2006.

A metrologia química no Inmetro. Disponível em: http://www.inmetro.gov.br. Acesso em: 9 mar. 2006.

DOQ-CGCRE-008: Orientação sobre validação de métodos de ensaios químicos. Revisão 02. Junho de 2007. Disponível em: http://www.inmetro.gov.br. Acesso em: 20 nov. 2008.
DOQ-CGCRE-016: Orientações para seleção e uso de materiais de referência. Revisão 01. Abril de 2008. Disponível em: http:// www.inmetro.gov.br. Acesso em: 20 nov. 2008

INTERNATIONAL LABORATORY ACCREDITATION COOPERATION - ILAC. Resolutions of the eighth ILAC General Assembly. ILAC Resolution GA 8.12. Disponível em: http:// www.ilac.org. Acesso em: 15 mai. 2007.

INTERNATIONAL ORGANIZATION FOR STANDARDIZATION - ISO. The role of reference materials: Achieving quality in analytical chemistry. 2000. Disponível em: http://www. iso.org. Acesso em: 16 mai. 2005.

ISO Guide 30: Terms and definitions used in connection with reference materials Genebra, 1992. 2. ed. 8 p.

ISO Guide 35: Reference materials General and statistical principles for certification. Genebra, 2006. 3.ed. 64 p.

MARCONI, M. de A.; LAKATOS, E. M. Técnicas de pesquisa. 7. ed. São Paulo: Atlas, 2008

McNAIR, B. ILAC Laboratory Survey on Conversion to ISO/IEC 17025. 2004. Disponível em http://www.ilac.org. Acesso em 05 dez.2008.

MOURA, S. S. Investigação do Processo de Utilização de Materiais de Referência: Estudo de Caso de Laboratórios de Análises de Águas da Natureza. Universidade Federal Fluminense, 2006. $219 \mathrm{p}$
NATIONAL ASSOCIATION OF TESTING AUTHORITIES - NATA. NATA Annual Report 2008. Disponível em: http://www.nata.asn.au. Acesso em 10 nov. 2008

NEVES, J. L. Pesquisa qualitativa: características, usos e possibilidades. Caderno de pesquisa em Administração, São Paulo, v. 1, n. 3, p, 1-5, $2 .^{\circ}$ sem. 1996

PARKANY, M.; KLICH, H.; RASBERRY, S. REMCO, the ISO Council Committee on Reference Materials - its first 25 years. Accreditation and quality assurance. Springer Verlag, 2001. (6) p. 226-235.

SILVA, G. M. P. Proposta para modificações no processo de acreditação de laboratórios da Coordenação Geral de Credenciamento do Instituto Nacional de Metrologia, Normalização e Qualidade Industrial. Universidade Federal Fluminense. Niterói, 2006. 164 p.

STEIGER, T.; PRADEL, R. Update on COMAR: the Internet database for certified reference materials. Accreditation and Quality Assurance. Springer Verlag, 2006. DOI 10.1007/s00769006-0234-5.

UNGER, P. S. The ILAC Arrangement - Part I. Accreditation and Quality Assurance. Springer Verlag, 2001. (6) p. 521-522.

VERGARA, S. C. Projetos e relatórios de pesquisa em administração. 6. ed. São Paulo: Atlas, 2005

\section{SOBRE OS AUTORES}

\section{Suzana Saboia de Moura}

Inmetro

End.: Rua Santa Alexandrina 416, $7^{\circ}$ andar - Rio Comprido - Rio de Janeiro - RJ - 20261-232

Cel: (21) 9738-7754 Fax (trabalho): (21) 2563-2860

E-mail: ssmoura@inmetro.gov.br e suzanamoura@terra.com.br

\section{Stella Regina Reis da Costa}

UFRRJ/IT/DTA

End.: BR 465, Km 7 - Seropédica - RJ - 23890-000

Cel: (21) 9802-3680 Fax: (21) 3787-3750

E-mail: stella@ufrrj.br e stellare@ig.com.br 